\title{
Correction to: An Inequality for Hölder Continuous Functions Generalizing a Result of Carlo Miranda
}

\author{
Massimo Lanza de Cristoforis
}

\section{Correction to:}

Chapter 10 in: C. Constanda (ed.), Computational and Analytic Methods

in Science and Engineering, Springer Proceedings in Complexity, https://doi.org/10.1007/978-3-030-48186-5_10

Unfortunately the family name of the author was incorrect in the online version and it has been corrected now so that the full name appears as follows in Springer web sites.

Massimo Lanza de Cristoforis 